\title{
On the maxima and sums of homogeneous Gaussian random fields *
}

\author{
Zhongquan Tan ${ }^{a, b \dagger}, \quad$ Linjun Tang ${ }^{b}$ \\ a. School of Mathematical Sciences, Zhejiang University, Hangzhou 310027, PR China; \\ b. College of Mathematics, Physics and Information Engineering, Jiaxing University, Jiaxing 314001, PR China
}

\begin{abstract}
Let $\left\{X_{\mathbf{n}}\right\}_{\mathbf{n} \geq \mathbf{1}}$ be a sequence of homogeneous Gaussian random field with mean 0 and variance 1. Under some conditions related to the correlation function of the field, the joint limiting distribution of the maximum and the sum of the Gaussian random field is obtained. The result is also extended to continuous time Gaussian random field.
\end{abstract}

Key Words: maxima; sums; Gaussian random field; weakly and strongly dependent

AMS Classification: Primary 62G70; secondary 60G60

\section{Introduction}

Let $\left\{X_{n}\right\}_{n \geq 1}$ be a sequence of random variables and denote by $M_{n}=\max \left\{X_{n}, 0 \leq k \leq n\right\}$ and $S_{n}=$ $\sum_{k=1}^{n} X_{k}$ the partial maximum and the partial sum, respectively. The joint limiting distributions of the partial maxima and partial sums of a sequence of random variables have been studied by many authors since they played important role in theoretical and applied probability. Chow and Teugels (1978) first studied the joint asymptotic behavior of partial sum and maximum for independent and identically distributed random variables. Anderson and Turkman (1991a,1991b) considered the strong mixing case under some additional conditions which was got rid of by Hsing (1995).

Based on the limit results for the maxima from Berman (1974) and Mittal and Ylvisaker (1975), many authors dealt with this problem for Gaussian sequences under some dependent conditions. Suppose that $\left\{X_{n}\right\}_{n \geq 1}$ is a sequence of stationary Gaussian random variables with correlation function $r_{n}$ satisfying the following condition:

$$
r_{n} \ln n \rightarrow \gamma \in[0, \infty] \text { as } n \rightarrow \infty \text {. }
$$

For $\gamma \in[0, \infty)$, Ho and Hsing (1996) and McCormick and Qi (2000) showed for any $x, y \in \mathbb{R}$,

$$
\lim _{n \rightarrow \infty} P\left(a_{n}\left(M_{n}-b_{n}\right) \leq x, \frac{S_{n}}{\sqrt{\operatorname{Var}\left(S_{n}\right)}} \leq y\right)=\int_{-\infty}^{y} \exp \left(-e^{-x-\gamma+\sqrt{2 \gamma} z}\right) \phi(z) d z
$$

where $\phi(\cdot)$ stands for the standard normal density function and

$$
a_{n}=(2 \ln n)^{1 / 2}, b_{n}=(2 \ln n)^{1 / 2}-(\ln \ln n+\ln 4 \pi) /\left(2(2 \ln n)^{1 / 2}\right) .
$$

For the case $\gamma=\infty$, if $r_{n}$ is convex with $r_{n}=o(1)$ and $\left(r_{n} \ln n\right)^{-1}$ is monotone with $\left(r_{n} \ln n\right)^{-1}=o(1)$, McCormick and Qi (2000) and Peng and Nadarajah (2002) proved that

$$
\lim _{n \rightarrow \infty} P\left(r_{n}^{1 / 2}\left(M_{n}-\left(1-r_{n}\right)^{1 / 2} b_{n}\right) \leq x, \frac{S_{n}}{\sqrt{\operatorname{Var}\left(S_{n}\right)}} \leq y\right)=\Phi(\min \{x, y\}) .
$$

\footnotetext{
${ }^{*}$ Research supported by National Science Foundation of China (No. 11501250), Natural Science Foundation of Zhejiang Province of China (No. LQ14A010012, LY15A010019), China Postdoctoral Science Foundation (No. 2016M600460) and Postdoctoral Research Program of Zhejiang Province(No. 507100-X81601).

${ }^{\dagger}$ Corresponding author. E-mail address: tzq728@163.com
}

(C) 2017. This manuscript version is made available under the Elsevier user license 
It is worth point out that the above conditions are provided by Mittal and Ylvisaker (1975) which derived the limit distribution of the maxima, and the convexity condition may be replaced by $r_{n}$ nonincreasing (see McCormick and Mittal (1976)) but at a considerable computational expense. In the literature condition (1) with $\gamma=0$ is referred to as the weak dependence or the Berman's condition and consequently the sequence $X$ is called a weakly dependent Gaussian sequence. In analogy, the sequence $X$ with correlation function satisfying condition (1) with $\gamma>0$ is called strongly dependent Gaussian sequence. For some recent work on the joint limiting distribution of partial sums and maxima for Gaussian sequences, we refer to Tan and Yang (2015) and the references therein. In this work, we are interested in the limit theorem for the maxima and partial sums of weakly and strongly dependent Gaussian random fields.

It is well known that Gaussian random fields play very important role in many applied sciences, such as, image analysis, atmospheric sciences, geostatistics, hydrology and agriculture, among others. See the monograph of Adler and Taylor (2007) for details.

Throughout this paper, denote the set of all positive integers and all non-negative integers by $\mathbb{Z}$ and $\mathbb{N}$, respectively. Let $\mathbb{Z}^{d}$ and $\mathbb{N}^{d}$ be the $d$-dimensional product spaces of $\mathbb{Z}$ and $\mathbb{N}$, respectively, where $d \geq 2$. In this paper, we only consider the case of $d=2$ since the results for higher dimensions follow analogous arguments. For $\mathbf{i}=\left(i_{1}, i_{2}\right)$ and $\mathbf{j}=\left(j_{1}, j_{2}\right), \mathbf{i} \leq \mathbf{j}, \mathbf{i}-\mathbf{j}$ and $\mathbf{i}^{\mathbf{j}}$ mean $i_{k} \leq j_{k}, k=1,2,\left(i_{1}-j_{1}, i_{2}-j_{2}\right)$ and $\left(i_{1}^{j_{1}}, i_{2}^{j_{2}}\right)$, respectively. $|\mathbf{i}|$ and $\mathbf{n} \rightarrow \infty$ mean $\left(\left|i_{1}\right|,\left|i_{2}\right|\right)$ and $n_{k} \rightarrow \infty, k=1,2$, respectively. Let $\mathbf{I}_{\mathbf{n}}=\left\{\mathbf{j} \in \mathbb{Z}^{2}\right.$ : $\left.1 \leq j_{i} \leq n_{i}, i=1,2\right\}$ and $\mathbf{J}_{\mathbf{n}}=\left\{\mathbf{j} \in \mathbb{N}^{2}: 0 \leq j_{i} \leq n_{i}, i=1,2\right\}$. Let $\chi_{\mathbf{E}}$ be the number of elements in $\mathbf{E}$ for any subset $\mathbf{E}$ of $\mathbb{Z}^{2}$. Let $\chi_{\mathbf{k}}=\prod_{i: k_{i} \neq 0}\left|k_{i}\right|$ for $\mathbf{k}=\left(k_{1}, k_{2}\right)$ and $\chi_{\mathbf{0}}=1$. Note that $\chi_{\mathbf{k}}=\chi_{\mathbf{I}_{\mathbf{k}}}$ when $\mathbf{k} \in \mathbb{Z}^{2}$. Let $\Phi(\cdot)$ and $\phi(\cdot)$ denote the cumulative distribution function of standard Gaussian random variable and its density function respectively.

Choi (2002) studied the extremes for stationary Gaussian random fields and obtained the Gumbel type extreme limit theorem. Pereira (2010) extended Choi's results to the non-stationary Gaussian random fields. The above mentioned work has been extended by Tan (2013) to more general cases.

Let $\left\{X_{\mathbf{n}}\right\}_{\mathbf{n} \geq \mathbf{1}}$ be a sequence of homogeneous Gaussian random field on $\mathbb{Z}^{2}$ with with mean 0 , variance 1 and covariance functions $r_{\mathbf{n}}=r_{\mathbf{j}, \mathbf{j}+\mathbf{n}}=E X_{\mathbf{j}} X_{\mathbf{j}+\mathbf{n}}$ satisfying the following conditions:

A1: $\lim _{\mathbf{n} \rightarrow \infty} r_{\mathbf{n}} \ln \chi_{\mathbf{n}}=r \in[0, \infty)$, both $r_{\left(n_{1}, 0\right)} \ln n_{1}$ and $r_{\left(0, n_{2}\right)} \ln n_{2}$ are bounded;

A2: $\left|r_{\mathbf{n}}\right|<1$ for $\mathbf{n} \neq \mathbf{0}$.

Under the above two conditions, Tan (2013) proved the following result.

Theorem 1.1. Let $\left\{X_{\mathbf{n}}\right\}_{\mathbf{n} \geq \mathbf{1}}$ be a sequence of homogeneous Gaussian random field on $\mathbb{Z}^{2}$ with mean 0 , variance 1. Assume that the covariance functions $r_{\mathbf{n}}$ satisfy $\mathbf{A 1}$ and $\mathbf{A 2}$. Then,

$$
\lim _{\mathbf{n} \rightarrow \infty} P\left(a_{\mathbf{n}}\left(M\left(\mathbf{I}_{\mathbf{n}}\right)-b_{\mathbf{n}}\right) \leq x\right)=\int_{-\infty}^{+\infty} \exp (-\exp (-x-r+\sqrt{2 r} z)) \phi(z) d z
$$

where $M\left(\mathbf{I}_{\mathbf{n}}\right)=\max _{\mathbf{i} \in \mathbf{I}_{\mathbf{n}}} X_{\mathbf{i}}, a_{\mathbf{n}}=\sqrt{2 \ln \chi_{\mathbf{n}}}$ and $b_{\mathbf{n}}=a_{\mathbf{n}}-\frac{\ln \ln \chi_{\mathbf{n}}+\ln (4 \pi)}{2 a_{\mathbf{n}}}$.

The case $r=\infty$ has been studied by McCormick (1978) and Mathew and McCormick (1993), but the conditions are not of the simple extensions of those provided by Mittal and Ylvisaker (1975). However, the isotropy will constrain us to a complicated class of correlation function and only a special class of convex isotropic covariance functions having an integral representation introduced by Mittal (1976) is tractable, see McCormick (1978) and Mathew and McCormick (1993) for more details. For further results concerning extremes in Gaussian random fields we refer the reader to Piterbarg (1996), Pereira (2010), Choi (2010), Tan and Wang (2014), Dȩbicki et al. (2015) and Pereira and Tan (2016). For other related results on random fields, we refer to Pereira and Ferreira (2006), Pereira (2009), Ferreira and Pereira (2012).

In this paper, we derive the joint limiting distribution of the maximum and the partial sum for the Gaussian random field which satisfies the conditions of Theorem 1.1. We also extend the result to continuous time Gaussian random field. The paper is organized as follows. Section 2 displays the main result. Some extensions of the main result is discussed in Section 3. All the proof are relegated to Sections 4. 


\section{Main result}

Now, we state our main result.

Theorem 2.1. Let $\left\{X_{\mathbf{n}}\right\}_{\mathbf{n} \geq \mathbf{1}}$ be a sequence of homogeneous Gaussian random field with mean 0, variance 1 . Assume that the covariance functions $r_{\mathbf{n}}=E X_{\mathbf{j}} X_{\mathbf{j}+\mathbf{n}}$ satisfy $\boldsymbol{A 1}$ and $\boldsymbol{A 2}$. Then,

$$
\lim _{\mathbf{n} \rightarrow \infty} P\left(a_{\mathbf{n}}\left(M\left(\mathbf{I}_{\mathbf{n}}\right)-b_{\mathbf{n}}\right) \leq x, \frac{S\left(\mathbf{I}_{\mathbf{n}}\right)}{\sqrt{\operatorname{Var}\left(S\left(\mathbf{I}_{\mathbf{n}}\right)\right)}} \leq y\right)=\int_{-\infty}^{y} \exp (-\exp (-x-r+\sqrt{2 r} z)) \phi(z) d z,
$$

where $S\left(\mathbf{I}_{\mathbf{n}}\right)=\sum_{\mathbf{k} \in \mathbf{I}_{\mathbf{n}}} X_{\mathbf{k}}$ and $a_{\mathbf{n}}, b_{\mathbf{n}}$ are define in Theorem 1.1.

Remark 2.1. (i). As in the one dimensional case, in the weakly dependent case, the maximum and the sum are asymptotically independent, while in the strongly dependent case, they are asymptotically dependent.

(ii). Letting $y \rightarrow \infty$ in Theorem 2.1, we get Theorem 1.1, so Theorem 2.1 is an extension of Theorem 1.1.

(iii). To study the case of $r=\infty$, we need more precise arguments on the covariance functions and this will be done based on the work of McCormick (1978) and Mathew and McCormick (1993) in a future paper.

\section{Extension}

In this section, we extend Theorem 2.1 to continuous time Gaussian random fields. Let $\{X(\mathbf{t}): \mathbf{t} \geq \mathbf{0}\}$ be a homogeneous Gaussian field with mean 0, variance 1 and covariance function

$$
r(\mathbf{t})=\operatorname{Cov}(X(\mathbf{t}), X(\mathbf{0}))
$$

We assume that the covariance function satisfies the following conditions:

B1: $r(\mathbf{t})=1-\left|t_{1}\right|^{\alpha_{1}}-\left|t_{2}\right|^{\alpha_{2}}+o\left(\left|t_{1}\right|^{\alpha_{1}}+\left|t_{2}\right|^{\alpha_{2}}\right)$ as $\mathbf{t} \rightarrow 0$ with $\alpha_{i} \in(0,2]$

B2: $r(\mathbf{t})<1$ for $\mathbf{t} \neq \mathbf{0}$;

B3: $\lim _{\mathbf{t} \rightarrow \infty} r(\mathbf{t}) \ln \chi_{\mathbf{t}}=r \in[0, \infty)$, both $r\left(t_{1}, 0\right) \ln t_{1}$ and $r\left(0, t_{2}\right) \ln t_{2}$ are bounded.

If conditions B1 and B2 hold, Theorem 7.1 of Piterbarg (1996) showed that for any fixed $\mathbf{h}>\mathbf{0}$

$$
P\left(\max _{\mathbf{t} \in\left[0, h_{1}\right] \times\left[0, h_{2}\right]} X(\mathbf{t})>u\right)=\mathcal{H}_{\alpha_{1}} \mathcal{H}_{\alpha_{2}} h_{1} h_{2} u^{2 / \alpha_{1}+2 / \alpha_{2}}(1-\Phi(u))(1+o(1)),
$$

as $u \rightarrow \infty$, where $\mathcal{H}_{\alpha_{i}}, i=1,2$ are the Pickands constants, which are defined by $\mathcal{H}_{\alpha}=\lim _{\lambda \rightarrow \infty} \mathcal{H}_{\alpha}(\lambda) / \lambda$, with

$$
\mathcal{H}_{\alpha}(\lambda)=E \exp \left(\max _{t \in[0, \lambda]} \sqrt{2} B_{\alpha / 2}(t)-t^{\alpha}\right)
$$

and $B_{H}$ a fractional Brownian motion, that is a Gaussian zero mean process with stationary increments such that $\mathbb{E} B_{H}^{2}(t)=|t|^{2 H}$. It is also well known that $0<\mathcal{H}_{\alpha}<\infty$, see e.g. Pickands (1969), Leadbetter et al. (1983) and Piterbarg (1996).

For $\mathbf{T} \geq \mathbf{0}$, denote $\mathbf{I}_{\mathbf{T}}=[\mathbf{0}, \mathbf{T}]$ and let

$$
M\left(\mathbf{I}_{\mathbf{T}}\right)=\max _{\mathbf{t} \in \mathbf{I}_{\mathbf{T}}} X(\mathbf{t}), \quad S\left(\mathbf{I}_{\mathbf{T}}\right)=\int_{\mathbf{I}_{\mathbf{T}}} X(\mathbf{t}) d \mathbf{t} .
$$

Theorem 3.1. Let $\{X(\mathbf{t}): \mathbf{t} \geq \mathbf{0}\}$ be a homogeneous Gaussian field with covariance function $r(\mathbf{t})$ satisfying B1, $\mathbf{B 2}$ and $\mathbf{B 3}$. Then we have

$$
\lim _{\mathbf{T} \rightarrow \infty} P\left(a_{\mathbf{T}}\left(M\left(\mathbf{I}_{\mathbf{T}}\right)-b_{\mathbf{T}}\right) \leq x, \frac{S\left(\mathbf{I}_{\mathbf{T}}\right)}{\sqrt{\operatorname{Var}\left(S\left(\mathbf{I}_{\mathbf{T}}\right)\right)}} \leq y\right)=\int_{-\infty}^{y} \exp (-\exp (-x-r+\sqrt{2 r} z)) \phi(z) d z,
$$

where $a_{\mathbf{T}}=\sqrt{2 \ln \chi_{\mathbf{T}}}, \quad b_{\mathbf{T}}=a_{\mathbf{T}}+a_{\mathbf{T}}^{-1} \ln \left((2 \pi)^{-1 / 2} \mathcal{H}_{\alpha_{1}} \mathcal{H}_{\alpha_{2}}\left(a_{\mathbf{T}}\right)^{1 / \alpha_{1}+1 / \alpha_{2}-1 / 2}\right)$. 


\section{Proofs}

In this section, we give the proofs of Theorem 2.1 and 3.1. We will split the proof of Theorems 2.1 and 3.1 into two cases, the weakly dependent case and the strongly dependent case. In the former case, we use some ideas from McCormick and Qi (2000), while in the latter case, we use some techniques from Ho and Hsing (1996). For the simplicity, let $u_{\mathbf{n}}=u_{\mathbf{n}}(x)=a_{\mathbf{n}}^{-1} x+b_{\mathbf{n}}$ and $u_{\mathbf{T}}=u_{\mathbf{T}}(x)=a_{\mathbf{T}}^{-1} x+b_{\mathbf{T}}$.

\subsection{Proof of Theorem 2.1}

We will prove that the maxima and the sum are asymptotically independent for the weakly dependent case. To that end, we will follow some procedures of McCormick and Qi (2000). Recall that $\mathbf{I}_{\mathbf{n}}=\left\{\mathbf{j} \in \mathbb{Z}^{2}: 1 \leq\right.$ $\left.j_{i} \leq n_{i}, i=1,2\right\}, \mathbf{J}_{\mathbf{n}}=\left\{\mathbf{j} \in \mathbb{N}^{2}: 0 \leq j_{i} \leq n_{i}, i=1,2\right\}, S\left(\mathbf{I}_{\mathbf{n}}\right)=\sum_{\mathbf{k} \in \mathbf{I}_{\mathbf{n}}} X_{\mathbf{k}}$ and $M\left(\mathbf{I}_{\mathbf{n}}\right)=\max _{\mathbf{k} \in \mathbf{I}_{\mathbf{n}}} X_{\mathbf{k}}$.

Noting that under the conditions $\mathbf{A} \mathbf{1}$ and $\mathbf{A} \mathbf{2}$ we have

$$
\lim _{\mathbf{n} \rightarrow \infty} \frac{\ln \chi_{\mathbf{n}}}{\chi_{\mathbf{n}}} \sum_{\mathbf{k} \in \mathbf{J}_{\mathbf{n}}}\left|r_{\mathbf{k}}\right|=0
$$

thus there exists a sequence $m(\mathbf{n})$ such that

$$
\lim _{\mathbf{n} \rightarrow \infty} m(\mathbf{n})=\infty
$$

and

$$
\lim _{\mathbf{n} \rightarrow \infty} \frac{m(\mathbf{n}) \ln \chi_{\mathbf{n}}}{\chi_{\mathbf{n}}} \sum_{\mathbf{k} \in \mathbf{J}_{\mathbf{n}}}\left|r_{\mathbf{k}}\right|=0
$$

Let

$$
\sigma_{\mathbf{n}}=\sqrt{\operatorname{Var}\left(S\left(\mathbf{I}_{\mathbf{n}}\right)\right)}, \quad \delta_{\mathbf{n}}(\mathbf{k})=E X_{\mathbf{k}} S\left(\mathbf{I}_{\mathbf{n}}\right)=\sum_{\mathbf{i} \in \mathbf{I}_{\mathbf{n}}} \frac{r_{\mathbf{k}-\mathbf{i}}}{\sigma_{\mathbf{n}}}, \quad \mathbf{k} \in \mathbf{I}_{\mathbf{n}}
$$

and define sets

$$
\mathbf{I}_{\mathbf{n}}^{+}=\left\{\mathbf{k}, \delta_{\mathbf{n}}(\mathbf{k}) \geq 0, \quad \mathbf{k} \in \mathbf{I}_{\mathbf{n}}\right\} \text { and } \mathbf{I}_{\mathbf{n}}^{-}=\left\{\mathbf{k}, \delta_{\mathbf{n}}(\mathbf{k})<0, \quad \mathbf{k} \in \mathbf{I}_{\mathbf{n}}\right\} .
$$

Note that $\mathbf{I}_{\mathbf{n}}^{+} \neq \emptyset$. Let

$$
s_{\mathbf{n}}^{+}=\sum_{\mathbf{k} \in \mathbf{I}_{\mathbf{n}}^{+}} \delta_{\mathbf{n}}(\mathbf{k}) \text { and } s_{\mathbf{n}}^{-}=\sum_{\mathbf{k} \in \mathbf{I}_{\mathbf{n}}^{-}} \delta_{\mathbf{n}}(\mathbf{k})
$$

Next define

$$
\mathbf{K}_{\mathbf{n}}^{+}=\left\{\mathbf{k} \in \mathbf{I}_{\mathbf{n}}^{+}: \frac{\delta_{\mathbf{n}}(\mathbf{k})}{s_{\mathbf{n}}^{+}} \geq \frac{\ln m(\mathbf{n})}{\chi_{\mathbf{n}}}\right\} \text { and } \mathbf{K}_{\mathbf{n}}^{-}=\left\{\mathbf{k} \in \mathbf{I}_{\mathbf{n}}^{-}: \frac{\delta_{\mathbf{n}}(\mathbf{k})}{s_{\mathbf{n}}^{-}} \geq \frac{\ln m(\mathbf{n})}{\chi_{\mathbf{n}}}\right\}
$$

Finally, set

$$
\mathbf{K}_{\mathbf{n}}=\mathbf{K}_{\mathbf{n}}^{+} \cup \mathbf{K}_{\mathbf{n}}^{-} \text {and } \mathbf{Q}_{\mathbf{n}}=\mathbf{I}_{\mathbf{n}} \backslash \mathbf{K}_{\mathbf{n}} .
$$

Lemma 4.1. For the $\mathbf{Q}_{\mathbf{n}}$ defined in (9), we have

$$
P\left\{\max _{\mathbf{k} \in \mathbf{Q}_{\mathbf{n}}} X_{\mathbf{k}} \leq u_{\mathbf{n}}\right\}-P\left\{\max _{\mathbf{k} \in \mathbf{I}_{\mathbf{n}}} X_{\mathbf{k}} \leq u_{\mathbf{n}}\right\} \leq P\left\{\max _{\mathbf{k} \in \mathbf{K}_{\mathbf{n}}} X_{\mathbf{k}} \geq u_{\mathbf{n}}\right\} \rightarrow 0
$$

as $\mathbf{n} \rightarrow \infty$.

Proof: By the stationarity of $X_{\mathbf{k}}$, we have

$$
P\left\{\max _{\mathbf{k} \in \mathbf{K}_{\mathbf{n}}} X_{\mathbf{k}} \geq u_{\mathbf{n}}\right\} \leq \sharp\left(\mathbf{K}_{\mathbf{n}}\right)\left(1-\Phi\left(u_{\mathbf{n}}\right)\right),
$$

where $\sharp(\cdot)$ denotes cardinality. Observe that

$$
\sharp\left(\mathbf{K}_{\mathbf{n}}^{+}\right) \frac{\ln m(\mathbf{n})}{\chi_{\mathbf{n}}} \leq \sum_{\mathbf{k} \in \mathbf{K}_{\mathbf{n}}^{+}} \frac{\delta_{\mathbf{n}}(\mathbf{k})}{s_{\mathbf{n}}^{+}} \leq 1
$$


and

$$
\sharp\left(\mathbf{K}_{\mathbf{n}}^{-}\right) \frac{\ln m(\mathbf{n})}{\chi_{\mathbf{n}}} \leq \sum_{\mathbf{k} \in \mathbf{K}_{\mathbf{n}}^{-}} \frac{\delta_{\mathbf{n}}(\mathbf{k})}{s_{\mathbf{n}}^{-}} \leq 1 .
$$

Therefore

$$
\sharp\left(\mathbf{K}_{\mathbf{n}}\right) \leq \frac{2 \chi_{\mathbf{n}}}{\ln m(\mathbf{n})} .
$$

Thus, we have

$$
P\left\{\max _{\mathbf{k} \in \mathbf{K}_{\mathbf{n}}} X_{\mathbf{k}} \geq u_{\mathbf{n}}\right\} \leq \frac{2 \chi_{\mathbf{n}}}{\ln m(\mathbf{n})}\left(1-\Phi\left(u_{\mathbf{n}}\right)\right)=o_{\mathbf{T}}(1)
$$

as $\mathbf{n} \rightarrow \infty$, since $\chi_{\mathbf{n}}\left(u_{\mathbf{n}}\right)^{2 / \alpha}\left(1-\Phi\left(u_{\mathbf{n}}\right)\right) \rightarrow e^{-x}$ as $\mathbf{n} \rightarrow \infty$ by the choice of $a_{\mathbf{n}}$ and $b_{\mathbf{n}}$. This completes the proof. $\square$

Next, we will produce an intermediary sequence sufficiently close to $X_{\mathbf{k}}$ but independent of $S_{\mathbf{n}}$. For $\mathbf{k} \in \mathbf{Q}_{\mathbf{n}}$ define constants $c_{\mathbf{n}}(\mathbf{k})$ by

$$
c_{\mathbf{n}}(\mathbf{k})=\frac{\delta_{\mathbf{n}}(\mathbf{k})}{s_{\mathbf{n}}^{+}}, \quad \mathbf{k} \in \mathbf{I}_{\mathbf{n}}^{+} \cap \mathbf{Q}_{\mathbf{n}}
$$

and if $I_{\mathbf{n}}^{-} \neq \emptyset$

$$
c_{\mathbf{n}}(\mathbf{k})=\frac{\delta_{\mathbf{n}}(\mathbf{k})}{s_{\mathbf{n}}^{-}}, \quad \mathbf{k} \in \mathbf{I}_{\mathbf{n}}^{-} \cap \mathbf{Q}_{\mathbf{n}}
$$

Define $\left\{Y_{\mathbf{k}}, \mathbf{k} \in \mathbf{Q}_{\mathbf{n}}\right\}$ by

$$
Y_{\mathbf{k}}=X_{\mathbf{k}}-c_{\mathbf{n}}(\mathbf{k}) S\left(\mathbf{I}_{\mathbf{n}}^{+}\right) \text {if } \mathbf{k} \in \mathbf{I}_{\mathbf{n}}^{+} \cap \mathbf{Q}_{\mathbf{n}}
$$

and

$$
Y_{\mathbf{k}}=X_{\mathbf{k}}-c_{\mathbf{n}}(\mathbf{k}) S\left(\mathbf{I}_{\mathbf{n}}^{-}\right) \text {if } \mathbf{k} \in \mathbf{I}_{\mathbf{n}}^{-} \cap \mathbf{Q}_{\mathbf{n}} .
$$

Lemma 4.2. For $\left\{Y_{\mathbf{k}}, \mathbf{k} \in \mathbf{Q}_{\mathbf{n}}\right\}$ defined as above, we have

$$
a_{\mathbf{n}}\left[\max _{\mathbf{k} \in \mathbf{Q}_{\mathbf{n}}} X_{\mathbf{k}}-\max _{\mathbf{k} \in \mathbf{Q}_{\mathbf{n}}} Y_{\mathbf{k}}\right] \stackrel{L^{1}}{\rightarrow} 0
$$

as $\mathbf{n} \rightarrow \infty$.

Proof: We have

$$
\begin{aligned}
\left|\max _{\mathbf{k} \in \mathbf{Q}_{\mathbf{n}}} X_{\mathbf{k}}-\max _{\mathbf{k} \in \mathbf{Q}_{\mathbf{n}}} Y_{\mathbf{k}}\right| & \leq \max _{\mathbf{k} \in \mathbf{Q}_{\mathbf{n}}}\left|X_{\mathbf{k}}-Y_{\mathbf{k}}\right| \\
& \leq \max _{\mathbf{k} \in \mathbf{Q}_{\mathbf{n}}}\left|c_{\mathbf{n}}(\mathbf{k})\right|\left[\left|S\left(\mathbf{I}_{\mathbf{n}}^{+}\right)\right|+\left|S\left(\mathbf{I}_{\mathbf{n}}^{-}\right)\right|\right] \\
& \leq \frac{\ln m(\mathbf{n})}{\chi_{\mathbf{n}}}\left[\left|S\left(\mathbf{I}_{\mathbf{n}}^{+}\right)\right|+\left|S\left(\mathbf{I}_{\mathbf{n}}^{-}\right)\right|\right] .
\end{aligned}
$$

Therefore using the above fact and Cauchy-Schwarz inequality

$$
\begin{aligned}
E\left|\max _{\mathbf{k} \in \mathbf{Q}_{\mathbf{n}}} X_{\mathbf{k}}-\max _{\mathbf{k} \in \mathbf{Q}_{\mathbf{n}}} Y_{\mathbf{k}}\right| & \leq \frac{\ln m(\mathbf{n})}{\chi_{\mathbf{n}}}\left[\operatorname{Var}^{1 / 2}\left(S\left(\mathbf{I}_{\mathbf{n}}^{+}\right)\right)+\operatorname{Var}^{1 / 2}\left(S\left(\mathbf{I}_{\mathbf{n}}^{-}\right)\right)\right] \\
& \leq 2 \frac{\ln m(\mathbf{n})}{\chi_{\mathbf{n}}}\left(\sum_{\mathbf{1} \leq \mathbf{k} \leq \mathbf{n}} \sum_{\mathbf{1} \leq \mathbf{l} \leq \mathbf{n}}|r(\mathbf{k}, \mathbf{l})|\right)^{1 / 2} \\
& \leq 2 \frac{\ln m(\mathbf{n})}{\chi_{\mathbf{n}}}\left(2 \chi_{\mathbf{n}} \sum_{\mathbf{1} \leq \mathbf{k} \leq \mathbf{n}}\left|r_{\mathbf{k}}\right|\right)^{1 / 2} \\
& =O(1) \frac{\ln m(\mathbf{n})}{\sqrt{m(\mathbf{n})}} \frac{1}{\sqrt{\ln \chi_{\mathbf{n}}}}\left(\frac{m(\mathbf{n}) \ln \chi_{\mathbf{n}}}{\chi_{\mathbf{n}}} \sum_{\mathbf{1} \leq \mathbf{k} \leq \mathbf{n}}\left|r_{\mathbf{k}}\right|\right)^{1 / 2} \\
& =o\left(a_{\mathbf{n}}^{-1}\right),
\end{aligned}
$$

which completes the proof of the lemma. $\square$

Proof of Theorem 2.1 for the weakly dependent case. We first observe that for $\mathbf{k} \in \mathbf{Q}_{\mathbf{n}}$,

$$
E\left(Y_{\mathbf{k}} S\left(\mathbf{I}_{\mathbf{n}}\right)\right)=0 .
$$


Therefore, since $\left(Y_{\mathbf{k}}, S\left(\mathbf{I}_{\mathbf{n}}\right)\right)$ have a joint normal distribution, the variables are independent. Now observe that

$$
\begin{aligned}
& \left|P\left\{\max _{\mathbf{k} \in \mathbf{I}_{\mathbf{n}}} X_{\mathbf{k}} \leq u_{\mathbf{n}}, S\left(\mathbf{I}_{\mathbf{n}}\right) / \sigma_{\mathbf{n}} \leq y\right\}-\exp \left\{-e^{-x}\right\} \Phi(y)\right| \\
& \leq\left|P\left\{\max _{\mathbf{k} \in \mathbf{I}_{\mathbf{n}}} X_{\mathbf{k}} \leq u_{\mathbf{n}}, S\left(\mathbf{I}_{\mathbf{n}}\right) / \sigma_{\mathbf{n}} \leq y\right\}-P\left\{\max _{\mathbf{k} \in \mathbf{Q}_{\mathbf{n}}} X_{\mathbf{k}} \leq u_{n}, S\left(\mathbf{I}_{\mathbf{n}}\right) / \sigma_{\mathbf{n}} \leq y\right\}\right| \\
& +\left|P\left\{\max _{\mathbf{k} \in \mathbf{Q}_{\mathbf{n}}} X_{\mathbf{k}} \leq u_{\mathbf{n}}, S\left(\mathbf{I}_{\mathbf{n}}\right) / \sigma_{\mathbf{n}} \leq y\right\}-P\left\{\max _{\mathbf{k} \in \mathbf{Q}_{\mathbf{n}}} Y_{\mathbf{k}} \leq u_{n}, S\left(\mathbf{I}_{\mathbf{n}}\right) / \sigma_{\mathbf{n}} \leq y\right\}\right| \\
& +\left|P\left\{\max _{\mathbf{k} \in \mathbf{Q}_{\mathbf{n}}} Y_{\mathbf{k}} \leq u_{\mathbf{n}}, S\left(\mathbf{I}_{\mathbf{n}}\right) / \sigma_{\mathbf{n}} \leq y\right\}-\exp \left\{-e^{-x}\right\} \Phi(y)\right| \\
& =: \sum_{i=1}^{3} W_{\mathbf{n} i} .
\end{aligned}
$$

By Lemma 4.1, it is easy to see that

$$
\begin{aligned}
W_{\mathbf{n} 1} & \leq P\left\{\max _{\mathbf{k} \in \mathbf{Q}_{\mathbf{n}}} X_{\mathbf{k}} \leq u_{\mathbf{n}}\right\}-P\left\{\max _{\mathbf{k} \in \mathbf{I}_{\mathbf{n}}} X_{\mathbf{k}} \leq u_{\mathbf{n}}\right\} \\
& \leq P\left\{\max _{\mathbf{k} \in \mathbf{K}_{\mathbf{n}}} X_{\mathbf{k}}>u_{\mathbf{n}}\right\} \rightarrow 0,
\end{aligned}
$$

as $\mathbf{n} \rightarrow \infty$. Using Lemma 4.2, Theorem 1.1 and (10), we have

$$
\begin{aligned}
W_{\mathbf{n} 2} & \leq E\left|I\left\{\max _{\mathbf{k} \in \mathbf{Q}_{\mathbf{n}}} Y_{\mathbf{k}} \leq u_{\mathbf{n}}\right\}-I\left\{\max _{\mathbf{k} \in \mathbf{Q}_{\mathbf{n}}} X_{\mathbf{k}} \leq u_{\mathbf{n}}\right\}\right| \\
& \leq P\left\{x-a_{\mathbf{n}}\left|\max _{\mathbf{k} \in \mathbf{Q}_{\mathbf{n}}} X_{\mathbf{k}}-\max _{\mathbf{k} \in \mathbf{Q}_{\mathbf{n}}} Y_{\mathbf{k}}\right| \leq a_{\mathbf{n}}\left(\max _{\mathbf{k} \in \mathbf{Q}_{\mathbf{n}}} X_{\mathbf{k}}-b_{\mathbf{n}}\right) \leq x+a_{\mathbf{n}}\left|\max _{\mathbf{k} \in \mathbf{Q}_{\mathbf{n}}} X_{\mathbf{k}}-\max _{\mathbf{k} \in \mathbf{Q}_{\mathbf{n}}} Y_{\mathbf{k}}\right|\right\} \\
& \rightarrow 0
\end{aligned}
$$

as $\mathbf{n} \rightarrow \infty$. For $W_{\mathbf{n} 3}$, we have by the independence, (10), (11) and Theorem 1.1

$$
\begin{aligned}
W_{\mathbf{n} 3}= & \Phi(y)\left|P\left\{\max _{\mathbf{k} \in \mathbf{Q}_{\mathbf{n}}} Y_{\mathbf{k}} \leq u_{\mathbf{n}}\right\}-\exp \left\{-e^{-x}\right\}\right| \\
\leq & \Phi(y)\left|P\left\{\max _{\mathbf{k} \in \mathbf{Q}_{\mathbf{n}}} Y_{\mathbf{k}} \leq u_{\mathbf{n}}\right\}-P\left\{\max _{\mathbf{k} \in \mathbf{Q}_{\mathbf{n}}} X_{\mathbf{k}} \leq u_{\mathbf{n}}\right\}\right| \\
& +\Phi(y)\left|P\left\{\max _{\mathbf{k} \in \mathbf{Q}_{\mathbf{n}}} X_{\mathbf{k}} \leq u_{\mathbf{n}}\right\}-P\left\{\max _{\mathbf{k} \in \mathbf{I}_{\mathbf{n}}} X_{\mathbf{k}} \leq u_{\mathbf{n}}\right\}\right| \\
& +\Phi(y)\left|P\left\{\max _{\mathbf{k} \in \mathbf{I}_{\mathbf{n}}} X_{\mathbf{k}} \leq u_{\mathbf{n}}\right\}-\exp \left\{-e^{-x}\right\}\right| \rightarrow 0
\end{aligned}
$$

as $\mathbf{n} \rightarrow \infty$. The proof of the theorem is complete.

Proof of Theorem $\mathbf{2 . 1}$ for the strongly dependent case. Let $\left\{Z_{\mathbf{n}}\right\}_{\mathbf{n} \geq \mathbf{1}}$ be an independent standardized Gaussian random field. Let

$$
\rho_{\mathbf{n}}=r / \ln \chi_{\mathbf{n}}
$$

and define

$$
\xi_{\mathbf{i}}=\sqrt{1-\rho_{\mathbf{n}}} Z_{\mathbf{i}}+\sqrt{\rho_{\mathbf{n}}} V, \quad \mathbf{i} \in \mathbf{I}_{\mathbf{n}},
$$

where $V$ is a standard Gaussian random variable independent of $\left\{Z_{\mathbf{n}}\right\}_{\mathbf{n} \geq \mathbf{1}}$. Note that the covariance function of $\left\{\xi_{\mathbf{i}}\right\}_{\mathbf{i} \in \mathbf{I}_{\mathbf{n}}}$ is equal to $\rho_{\mathbf{n}}$. Let $M_{\xi}\left(\mathbf{I}_{\mathbf{n}}\right)=\max _{\mathbf{i} \in \mathbf{I}_{\mathbf{n}}} \xi_{\mathbf{i}}$ and $\widehat{r}_{\mathbf{k}, \mathbf{n}}=E\left(X_{\mathbf{k}} S\left(\mathbf{I}_{\mathbf{n}}\right) / \sigma_{\mathbf{n}}\right)$.

Firstly, we prove that

$$
\left|P\left(M\left(\mathbf{I}_{\mathbf{n}}\right) \leq u_{\mathbf{n}}, S\left(\mathbf{I}_{\mathbf{n}}\right) / \sigma_{\mathbf{n}} \leq y\right)-P\left(M_{\xi}\left(\mathbf{I}_{\mathbf{n}}\right) \leq u_{\mathbf{n}}, V \leq y\right)\right| \rightarrow 0,
$$

as $\mathbf{n} \rightarrow \infty$. By the Normal Comparison Lemma (see Leadbetter et al. (1983) and Piterbarg (1996)), we have

$$
\left|P\left(M\left(\mathbf{I}_{\mathbf{n}}\right) \leq u_{\mathbf{n}}, S\left(\mathbf{I}_{\mathbf{n}}\right) / \sigma_{\mathbf{n}} \leq y\right)-P\left(M_{\xi}\left(\mathbf{I}_{\mathbf{n}}\right) \leq u_{\mathbf{n}}, V \leq y\right)\right|
$$




$$
\begin{aligned}
& \leq \sum_{\mathbf{k} \neq \mathbf{l} \in \mathbf{I}_{\mathbf{n}}}\left|r_{\mathbf{k}, \mathbf{l}}-\rho_{\mathbf{n}}\right| \int_{0}^{1} \phi\left(u_{\mathbf{n}}, u_{\mathbf{n}}, \theta_{\mathbf{k}, \mathbf{l}}^{(h)}\right) d h+\sum_{\mathbf{k} \in \mathbf{I}_{\mathbf{n}}}\left|\widehat{r}_{\mathbf{k}, \mathbf{n}}-\sqrt{\rho_{\mathbf{n}}}\right| \int_{0}^{1} \phi\left(u_{\mathbf{n}}, y, \vartheta_{\mathbf{k}, \mathbf{n}}^{(h)}\right) d h \\
& =: \Sigma_{\mathbf{n}, 1}+\Sigma_{\mathbf{n}, 2} \text {, }
\end{aligned}
$$

where $\theta_{\mathbf{k}, \mathbf{l}}^{(h)}=h r_{\mathbf{k}, \mathbf{l}}+(1-h) \rho_{\mathbf{n}}, \vartheta_{\mathbf{k}, \mathbf{n}}^{(h)}=h \widehat{r}_{\mathbf{k}, \mathbf{n}}+(1-h) \sqrt{\rho_{\mathbf{n}}}$ and $\phi(x, y, r)$ stands for the two dimensional normal density function, i.e.,

$$
\phi(x, y, r)=\frac{1}{2 \pi \sqrt{1-r^{2}}} \exp \left(-\frac{x^{2}-2 r x y+y^{2}}{2\left(1-r^{2}\right)}\right) .
$$

By some computations and Lemma 3.2 of Tan (2013), we have for some constant $K>0$

$$
\Sigma_{\mathbf{n}, 1} \leq K \chi_{\mathbf{n}} \sum_{\mathbf{k} \in \mathbf{J}_{\mathbf{n}}, \mathbf{k} \neq \mathbf{0}}\left|r_{\mathbf{k}}-\rho_{\mathbf{n}}\right| \exp \left(-\frac{u_{\mathbf{n}}^{2}}{1+\omega_{\mathbf{k}}}\right) \rightarrow 0,
$$

as $\mathbf{n} \rightarrow \infty$. Now, to prove (12), it suffices to show that $\Sigma_{\mathbf{n}, 2} \rightarrow 0$ as $\mathbf{n} \rightarrow \infty$. For simplicity, write $\vartheta$ for $\vartheta_{\mathbf{k}, \mathbf{n}}^{(h)}$. Since $x e^{-x}$ is decreasing on $(1, \infty)$ and for large $\mathbf{n} \sup _{\mathbf{k} \in \mathbf{I}_{\mathbf{n}}}\left|\vartheta_{\mathbf{k}, \mathbf{n}}^{(h)}\right| \leq \varepsilon<1$, we have that for large $\mathbf{n}$

$$
\begin{aligned}
& \frac{u_{\mathbf{n}}^{2}-2 \vartheta y u_{\mathbf{n}}+y^{2}}{2\left(1-\vartheta^{2}\right)} \exp \left(-\frac{u_{\mathbf{n}}^{2}-2 \vartheta y u_{\mathbf{n}}+y^{2}}{2\left(1-\vartheta^{2}\right)}\right) \\
& \leq \frac{u_{\mathbf{n}}^{2}-2 \vartheta y u_{\mathbf{n}}+y^{2}}{2} \exp \left(-\frac{u_{\mathbf{n}}^{2}-2 \vartheta y u_{\mathbf{n}}+y^{2}}{2}\right)
\end{aligned}
$$

and hence that

$$
\begin{aligned}
& \frac{1}{\sqrt{1-\vartheta^{2}}} \exp \left(-\frac{u_{\mathbf{n}}^{2}-2 \vartheta y u_{\mathbf{n}}+y^{2}}{2\left(1-\vartheta^{2}\right)}\right) \\
& =\frac{2 \sqrt{1-\vartheta^{2}}}{u_{\mathbf{n}}^{2}-2 \vartheta y u_{\mathbf{n}}+y^{2}} \frac{u_{\mathbf{n}}^{2}-2 \vartheta y u_{\mathbf{n}}+y^{2}}{2\left(1-\vartheta^{2}\right)} \exp \left(-\frac{u_{\mathbf{n}}^{2}-2 \vartheta y u_{\mathbf{n}}+y^{2}}{2(1-\vartheta)}\right) \\
& \leq \exp \left(-\frac{u_{\mathbf{n}}^{2}-2 \vartheta y u_{\mathbf{n}}+y^{2}}{2}\right) .
\end{aligned}
$$

Thus for $\mathbf{n}$ large enough

$$
\Sigma_{n, 2} \leq \sum_{\mathbf{k} \in \mathbf{I}_{\mathbf{n}}}\left|\widehat{r}_{\mathbf{k}, \mathbf{n}}-\sqrt{\rho_{\mathbf{n}}}\right| \int_{0}^{1} \exp \left(-\frac{u_{\mathbf{n}}^{2}-2 \vartheta y u_{\mathbf{n}}+y^{2}}{2}\right) d h
$$

Now we assume that $y \neq 0$. The case $y=0$ can be handled by a continuity argument or by slightly modifying the proof below. Since $e^{-u_{\mathbf{n}}^{2} / 2} \sim 2 \sqrt{\pi} e^{-x}\left(\sqrt{\ln \chi_{\mathbf{n}}} / \chi_{\mathbf{n}}\right), u_{\mathbf{n}} \sim \sqrt{2 \ln \chi_{\mathbf{n}}}$ and

$$
\int_{0}^{1} \exp \left(\vartheta y u_{\mathbf{n}}\right) d h=\exp \left(\sqrt{\rho_{\mathbf{n}}} y u_{\mathbf{n}}\right)\left|\frac{\exp \left\{\left[\widehat{r}_{\mathbf{k}, \mathbf{n}}-\left(\rho_{\mathbf{n}}\right)^{1 / 2}\right] y u_{\mathbf{n}}\right\}-1}{\left[\widehat{r}_{\mathbf{k}, \mathbf{n}}-\left(\rho_{\mathbf{n}}\right)^{1 / 2}\right] y u_{\mathbf{n}}}\right|,
$$

we have for some constants $\mathcal{C}>0$

$$
\Sigma_{\mathbf{n}, 2} \leq \mathcal{C} \frac{\exp \left(-x+\sqrt{2 r} y-y^{2} / 2\right)}{|y|} \frac{1}{\chi_{\mathbf{n}}} \sum_{\mathbf{k} \in \mathbf{I}_{\mathbf{n}}}\left|\exp \left\{\left[\widehat{r}_{\mathbf{k}, \mathbf{n}}-\left(\rho_{\mathbf{n}}\right)^{1 / 2}\right] y u_{\mathbf{n}}\right\}-1\right| .
$$

Consequently, to show that $\Sigma_{\mathbf{n}, 2} \rightarrow 0$ as $\mathbf{n} \rightarrow \infty$, it suffices to show that for some sequence $\varepsilon_{\mathbf{n}} \in(0,1)$ which tends to 1 and such that $\mathbf{n}^{\varepsilon_{\mathbf{n}}-1} \rightarrow(0,0)$, we have

$$
\max _{\mathbf{k} \in\left[\mathbf{n}^{\left.\varepsilon_{\mathbf{n}}, \mathbf{n}-\mathbf{n}^{\varepsilon} \mathbf{n}\right]}\right.}\left[\widehat{r}_{\mathbf{k}, \mathbf{n}}-\left(\rho_{\mathbf{n}}\right)^{1 / 2}\right] \sqrt{\ln \chi_{\mathbf{n}}} \rightarrow 0,
$$

as $\mathbf{n} \rightarrow \infty$. First, note that $\sqrt{\rho_{\mathbf{n}} \ln \chi_{\mathbf{n}}}=\sqrt{r}$ and write

$$
\widehat{r}_{\mathbf{k}, \mathbf{n}}=E\left(X_{\mathbf{k}} \frac{S_{\mathbf{n}}}{\sigma_{\mathbf{n}}}\right)=\frac{\sum_{\mathbf{l} \in \mathbf{I}_{\mathbf{n}}} r_{\mathbf{k}, \mathbf{l}}}{\sqrt{\sum_{\mathbf{k}, \mathbf{l} \in \mathbf{I}_{\mathbf{n}}} r_{\mathbf{k}, \mathbf{l}}}}
$$




$$
=\frac{\sum_{\mathbf{l} \in \mathbf{J}_{\mathbf{k}-1}} r_{\mathbf{l}}+\sum_{\mathbf{l} \in \mathbf{I}_{\mathbf{n}-\mathbf{k}}} r_{\mathbf{l}}}{\sqrt{\sum_{\mathbf{k} \in \mathbf{I}_{\mathbf{n}}}\left[\sum_{\mathbf{l} \in \mathbf{J}_{\mathbf{k}-1}} r_{\mathbf{l}}+\sum_{\mathbf{l} \in \mathbf{I}_{\mathbf{n}-\mathbf{k}}} r_{\mathbf{l}}\right]}} .
$$

Using the simple facts that as $\mathbf{n} \rightarrow \infty$

$$
\sum_{\mathbf{k} \in \mathbf{I}_{\mathbf{n}}} \frac{r}{\ln \chi_{\mathbf{k}}} \sim r \frac{\chi_{\mathbf{n}}}{\ln \chi_{\mathbf{n}}}, \quad \sum_{\mathbf{k} \in \mathbf{I}_{\mathbf{n}}} \frac{\chi_{\mathbf{k}} r}{\ln \chi_{\mathbf{k}}} \sim \frac{r}{2} \frac{\left(\chi_{\mathbf{n}}\right)^{2}}{\ln \chi_{\mathbf{n}}}
$$

we conclude readily from condition $\mathbf{A} \mathbf{1}$ that, uniformly for $\mathbf{k} \in\left[\mathbf{n}^{\varepsilon_{\mathbf{n}}}, \mathbf{n}-\mathbf{n}^{\varepsilon_{\mathbf{n}}}\right]$

$$
\sum_{\mathbf{l} \in \mathbf{J}_{\mathbf{k}-\mathbf{1}}} r_{\mathbf{l}}+\sum_{\mathbf{l} \in \mathbf{I}_{\mathbf{n}-\mathbf{k}}} r_{\mathbf{l}} \sim \sum_{\mathbf{l} \in \mathbf{J}_{\mathbf{k}-\mathbf{1}}} \frac{r}{\ln \chi_{\mathbf{l}}}+\sum_{\mathbf{l} \in \mathbf{I}_{\mathbf{n}-\mathbf{k}}} \frac{r}{\ln \chi_{\mathbf{l}}} \sim r \frac{\chi_{\mathbf{n}}}{\ln \chi_{\mathbf{n}}},
$$

as $\mathbf{n} \rightarrow \infty$. Using fact (15) and conditions $\mathbf{A 1}$ and $\mathbf{A 2}$ again, we have as $\mathbf{n} \rightarrow \infty$

$$
\sum_{\mathbf{k} \in \mathbf{I}_{\mathbf{n}}} \sum_{\mathbf{l} \in \mathbf{J}_{\mathbf{k}-\mathbf{1}}} r_{\mathbf{l}} \sim \sum_{\mathbf{k} \in \mathbf{I}_{\mathbf{n}}} r \frac{\chi_{\mathbf{k}}}{\ln \chi_{\mathbf{k}}} \sim \frac{r}{2} \frac{\left(\chi_{\mathbf{n}}\right)^{2}}{\ln \chi_{\mathbf{n}}}
$$

and

$$
\sum_{\mathbf{k} \in \mathbf{I}_{\mathbf{n}}} \sum_{\mathbf{l} \in \mathbf{I}_{\mathbf{n}-\mathbf{k}}} r_{\mathbf{l}} \sim \sum_{\mathbf{k} \in \mathbf{I}_{\mathbf{n}}} r \frac{\chi_{\mathbf{n}-\mathbf{k}}}{\ln \chi_{\mathbf{n}-\mathbf{k}}} \sim \frac{r}{2} \frac{\left(\chi_{\mathbf{n}}\right)^{2}}{\ln \chi_{\mathbf{n}}}
$$

Thus we conclude that as $\mathbf{n} \rightarrow \infty$

$$
\widehat{r}_{\mathbf{k}, \mathbf{n}} \sqrt{\ln \chi_{\mathbf{n}}} \rightarrow \sqrt{r}
$$

Thus we get that $\Sigma_{\mathbf{n}, 2} \rightarrow 0$ as $\mathbf{n} \rightarrow \infty$.

Secondly, in view of the definition of $\left\{\xi_{\mathbf{i}}\right\}_{\mathbf{i} \in \mathbf{I}_{\mathbf{n}}}$, we have

$$
\begin{aligned}
P\left(M_{\xi}\left(\mathbf{I}_{\mathbf{n}}\right) \leq u_{\mathbf{n}}, V \leq y\right) & =P\left(\max _{\mathbf{i} \in \mathbf{I}_{\mathbf{n}}} \xi_{\mathbf{i}} \leq u_{\mathbf{n}}, V \leq y\right) \\
& =P\left(\sqrt{1-\rho_{\mathbf{n}}} \max _{\mathbf{i} \in \mathbf{I}_{\mathbf{n}}} Z_{\mathbf{i}}+\sqrt{\rho_{\mathbf{n}}} V \leq u_{\mathbf{n}}, V \leq y\right) \\
& =\int_{-\infty}^{y} P\left(\sqrt{1-\rho_{\mathbf{n}}} \max _{\mathbf{i} \in \mathbf{I}_{\mathbf{n}}} Z_{\mathbf{i}}+\sqrt{\rho_{\mathbf{n}}} z \leq u_{\mathbf{n}}\right) \phi(z) d z \\
& =\int_{-\infty}^{y} P\left(\max _{\mathbf{i} \in \mathbf{I}_{\mathbf{n}}} Z_{\mathbf{i}} \leq \frac{u_{\mathbf{n}}-\sqrt{\rho_{\mathbf{n}}} z}{\sqrt{1-\rho_{\mathbf{n}}}}\right) \phi(z) d z .
\end{aligned}
$$

It is easy to check that as $\mathbf{n} \rightarrow \infty$

$$
\begin{aligned}
u_{\mathbf{n}}^{(z)} & :=\frac{u_{\mathbf{n}}-\sqrt{\rho_{n}} z}{\sqrt{1-\rho_{\mathbf{n}}}}=\left(u_{\mathbf{n}}-\sqrt{\rho_{\mathbf{n}}} z\right)\left(1+\frac{1}{2} \rho_{\mathbf{n}}+o\left(\rho_{\mathbf{n}}\right)\right) \\
& =u_{\mathbf{n}}+\frac{r-\sqrt{2 r} z}{u_{\mathbf{n}}}+o\left(u_{\mathbf{n}}^{-1}\right) .
\end{aligned}
$$

Thus, it follows from $\lim _{\mathbf{n} \rightarrow \infty} \chi_{\mathbf{n}}\left(1-\Phi\left(u_{\mathbf{n}}\right)\right)=e^{-x}$ that

$$
\lim _{\mathbf{n} \rightarrow \infty} \chi_{\mathbf{n}}\left(1-\Phi\left(u_{\mathbf{n}}^{(z)}\right)\right)=e^{-x-r+\sqrt{2 r} z} .
$$

Now, noting that $\mathbf{Z}=\left\{Z_{\mathbf{n}}\right\}_{\mathbf{n} \geq 1}$ is an independent standardized Gaussian random field, we have

$$
P\left(\max _{\mathbf{i} \in \mathbf{I}_{\mathbf{n}}} Z_{\mathbf{i}} \leq u_{\mathbf{n}}^{(z)}\right)=\prod_{\mathbf{i} \in \mathbf{I}_{\mathbf{n}}} P\left(Z_{\mathbf{i}} \leq u_{\mathbf{n}}^{(z)}\right)=\chi_{\mathbf{n}}\left(1-\Phi\left(u_{\mathbf{n}}^{(z)}\right)\right)=e^{-x-r+\sqrt{2 r} z}(1+o(1))
$$

as $\mathbf{n} \rightarrow \infty$. Combining the above result with (12),(16) and applying the dominated convergence theorem we obtain the desired result. 


\subsection{Proof of Theorem 3.1}

To prove Theorem 3.1, the continuous set $\mathbf{I}_{\mathbf{T}}=[\mathbf{0}, \mathbf{T}]$ should be discretised. So, we introduce the uniform grids $\mathfrak{R}\left(q_{i}\right)=\left\{k q_{i}: k=0,1,2, \cdots\right\}, q_{i}>0, i=1,2$, where $q_{i}$ satisfies for some $\varepsilon>0$

$$
q_{i}=\varepsilon\left(2 \ln T_{i}\right)^{1 / \alpha_{i}} .
$$

For simplicity, let $\sigma_{\mathbf{T}}=\operatorname{Var}\left(S\left(\mathbf{I}_{\mathbf{T}}\right)\right)$. The following lemma plays an important role in the proof of Theorem 3.1 .

Lemma 4.3. Assume that $\mathbf{A 1}$ and $\mathbf{A 2}$ hold. Then for rectangle $\mathbf{I}_{\mathbf{h}}=[\mathbf{0}, \mathbf{h}]$ with fixed $\mathbf{h}>\mathbf{0}$, we have

$$
\left|P\left(\max _{\mathbf{t} \in \mathbf{I}_{\mathbf{h}} \cap \Re\left(q_{1}\right) \times \Re\left(q_{2}\right)} X(\mathbf{t}) \leq u_{\mathbf{T}}\right)-P\left(\max _{\mathbf{t} \in \mathbf{I}_{\mathbf{h}}} X(\mathbf{t}) \leq u_{\mathbf{T}}\right)\right| \leq h_{1} h_{2} \kappa(\varepsilon) u_{\mathbf{T}}^{2 / \alpha_{1}+2 / \alpha_{2}}\left(1-\Phi\left(u_{\mathbf{T}}\right)\right)(1+o(1)),
$$

where $\kappa(\varepsilon) \rightarrow 0$ as $\varepsilon \downarrow 0$.

Proof: See Lemma 1 of Dębicki et al. (2015). $\square$

Proof of Theorem $\mathbf{3 . 1}$ for the weakly dependent case. Using Lemma 4.3, it is easy to show the that

$$
\left|P\left(\max _{\mathbf{t} \in \mathbf{I}_{\mathbf{T}} \cap \mathfrak{R}\left(q_{1}\right) \times \Re\left(q_{2}\right)} X(\mathbf{t}) \leq u_{\mathbf{T}}, S\left(\mathbf{I}_{\mathbf{T}}\right) / \sigma_{\mathbf{T}} \leq y\right)-P\left(\max _{\mathbf{t} \in \mathbf{I}_{\mathbf{T}}} X(\mathbf{t}) \leq u_{\mathbf{T}}, S\left(\mathbf{I}_{\mathbf{T}}\right) / \sigma_{\mathbf{T}} \leq y\right)\right| \rightarrow 0,
$$

as $\varepsilon \downarrow 0$. Thus, to establish the result it suffices to show that

$$
\lim _{\varepsilon \downarrow 0} \lim _{\mathbf{T} \rightarrow \infty} P\left(\max _{\mathbf{t} \in \mathbf{I}_{\mathbf{T}} \cap \mathfrak{R}\left(q_{1}\right) \times \Re\left(q_{2}\right)} X(\mathbf{t}) \leq u_{\mathbf{T}}, S\left(\mathbf{I}_{\mathbf{T}}\right) / \sigma_{\mathbf{T}} \leq y\right)=\exp \left(-e^{-x}\right) \Phi(y) .
$$

Applying first Theorem 2.1, we get that asymptotics independence and then letting $\varepsilon \downarrow 0$, we obtain the desired result.

To deal with the strongly dependent case, divide $\left[0, T_{i}\right]$ into intervals with length $\delta$ alternating with shorter intervals with length $1-\delta$ for some constant $\delta>0$ and denote the long intervals by $S_{k}^{(i)}, k=1,2, \cdots, n_{i}=\left\lfloor T_{i}\right\rfloor$, where $\lfloor x\rfloor$ denotes the integral part of $x$. Denote $\mathbf{S}=\cup \mathbf{S}_{\mathbf{i}}=\cup S_{i_{1}}^{(1)} \times \cup S_{i_{2}}^{(2)}, \mathbf{R}=\mathbf{I}_{\mathbf{T}} \backslash \mathbf{S}$. It will be show blow that the area $\mathbf{R}$ plays no role in our consideration.

Let $\left\{X_{\mathbf{i}}(\mathbf{t}), \mathbf{t} \geq \mathbf{0}\right\}, \mathbf{i} \geq \mathbf{1}$ be independent copies of $\{X(\mathbf{t}), \mathbf{t} \geq \mathbf{0}\}$ and $\{\eta(\mathbf{t}), \mathbf{t} \geq \mathbf{0}\}$ be such that $\eta(\mathbf{t})=X_{\mathbf{i}}(\mathbf{t})$ for $\mathbf{t} \in \mathbf{E}_{\mathbf{i}}=\left[i_{1}-1, i_{1}\right) \times\left[i_{2}-1, i_{2}\right)$. Denote by $\gamma(\mathbf{s}, \mathbf{t})$ the covariance function of $\{\eta(\mathbf{t}), \mathbf{t} \geq \mathbf{0}\}$. It is easy to see that

$$
\gamma(\mathbf{s}, \mathbf{t})=\left\{\begin{array}{cl}
r(\mathbf{t}, \mathbf{s}), & \mathbf{s} \in \mathbf{E}_{\mathbf{i}}, \mathbf{t} \in \mathbf{E}_{\mathbf{j}}, \mathbf{i}=\mathbf{j} \\
0, & \mathbf{s} \in \mathbf{E}_{\mathbf{i}}, \mathbf{t} \in \mathbf{E}_{\mathbf{j}}, \mathbf{i} \neq \mathbf{j}
\end{array}\right.
$$

Define

$$
\xi_{\mathbf{T}}(\mathbf{t})=(1-\rho(\mathbf{T}))^{1 / 2} \eta(\mathbf{t})+\rho^{1 / 2}(\mathbf{T}) U, \quad \mathbf{t} \in \mathbf{I}_{\mathbf{T}},
$$

where $\rho(\mathbf{T})=r / \ln \chi_{\mathbf{T}}$ and $U$ is a standard normal random variable which is independent of $\{\eta(\mathbf{t}), \mathbf{t} \geq \mathbf{0}\}$. Denote by $\varrho(\mathbf{s}, \mathbf{t})$ the covariance function of $\left\{\xi_{\mathbf{T}}(\mathbf{t}), \mathbf{t} \in \mathbf{I}_{\mathbf{T}}\right\}$. It is easy to check that

$$
\varrho(\mathbf{s}, \mathbf{t})=\left\{\begin{array}{cl}
r(\mathbf{t}, \mathbf{s})+(1-r(\mathbf{t}, \mathbf{s})) \rho(\mathbf{T}), & \mathbf{s} \in \mathbf{E}_{\mathbf{i}}, \mathbf{t} \in \mathbf{E}_{\mathbf{j}}, \mathbf{i}=\mathbf{j} \\
\rho(\mathbf{T}), & \mathbf{s} \in \mathbf{E}_{\mathbf{i}}, \mathbf{t} \in \mathbf{E}_{\mathbf{j}}, \mathbf{i} \neq \mathbf{j}
\end{array}\right.
$$

Proof of Theorem 3.1 for the strongly dependent case. Firstly, by the same arguments as in the proof of Lemma 3.1 of Tan and Wang (2015), we can show that as $\delta \rightarrow 0$

$$
\left|P\left(a_{\mathbf{T}}\left(M\left(\mathbf{I}_{\mathbf{T}}\right)-b_{\mathbf{T}}\right) \leq x, S\left(\mathbf{I}_{\mathbf{T}}\right) / \sigma_{\mathbf{T}} \leq y\right)-P\left(a_{\mathbf{T}}\left(\max _{\mathbf{t} \in \mathbf{S}} X(\mathbf{t})-b_{\mathbf{T}}\right) \leq x, S\left(\mathbf{I}_{\mathbf{T}}\right) / \sigma_{\mathbf{T}} \leq y\right)\right| \rightarrow 0 .
$$

Secondly, by Lemma 4.3, we have

$$
\mid P\left(a_{\mathbf{T}}\left(\max _{\mathbf{t} \in \mathbf{S}} X(\mathbf{t})-b_{\mathbf{T}}\right) \leq x, S\left(\mathbf{I}_{\mathbf{T}}\right) / \sigma_{\mathbf{T}} \leq y\right)
$$




$$
-P\left(a_{\mathbf{T}}\left(\max _{\mathbf{t} \in \mathfrak{R}\left(q_{1}\right) \times \Re\left(q_{2}\right) \cap \mathbf{S}} X(\mathbf{t})-b_{T}\right) \leq x, S\left(\mathbf{I}_{\mathbf{T}}\right) / \sigma_{\mathbf{T}} \leq y\right) \mid \rightarrow 0
$$

as $\mathbf{T} \rightarrow \infty$ and $\varepsilon \downarrow 0$.

Thirdly, we show that

$$
\begin{aligned}
& \mid P\left(a_{\mathbf{T}}\left(\max _{\mathbf{t} \in \mathfrak{R}\left(q_{1}\right) \times \mathfrak{R}\left(q_{2}\right) \cap \mathbf{S}} X(\mathbf{t})-b_{T}\right) \leq x, S\left(\mathbf{I}_{\mathbf{T}}\right) / \sigma_{\mathbf{T}} \leq y\right) \\
& -P\left(a_{\mathbf{T}}\left(\max _{\mathbf{t} \in \mathfrak{R}\left(q_{1}\right) \times \mathfrak{R}\left(q_{2}\right) \cap \mathbf{S}} \xi_{\mathbf{T}}(\mathbf{t})-b_{\mathbf{T}}\right) \leq x, U \leq y\right) \mid \rightarrow 0
\end{aligned}
$$

uniformly for $\varepsilon>0$, as $\mathbf{T} \rightarrow \infty$.

Let $\widehat{r}(\mathbf{k q}, \mathbf{T})=E\left(X(\mathbf{k q}) S\left(\mathbf{I}_{\mathbf{T}}\right) / \sigma_{\mathbf{T}}\right)$. By Normal Comparison Lemma again, we have

$$
\begin{aligned}
& \mid P\left(a_{\mathbf{T}}\left(\max _{\mathbf{t} \in \mathfrak{R}\left(q_{1}\right) \times \mathfrak{R}\left(q_{2}\right) \cap \mathbf{S}} X(\mathbf{t})-b_{T}\right) \leq x, S\left(\mathbf{I}_{\mathbf{T}}\right) / \sigma_{\mathbf{T}} \leq y\right) \\
& -P\left(a_{\mathbf{T}}\left(\max _{\mathbf{t} \in \mathfrak{R}\left(q_{1}\right) \times \mathfrak{R}\left(q_{2}\right) \cap \mathbf{S}} \xi_{\mathbf{T}}(\mathbf{t})-b_{\mathbf{T}}\right) \leq x, U \leq y\right) \\
& \leq \sum_{\mathbf{k} \mathbf{q} \neq \mathbf{l} \mathbf{q} \in \mathbf{S}}|r(\mathbf{k q}, \mathbf{l q})-\varrho(\mathbf{k q}, \mathbf{l q})| \int_{0}^{1} \phi\left(u_{\mathbf{T}}, u_{\mathbf{T}}, \theta_{\mathbf{k}, \mathbf{l}}^{(h)}\right) d h \\
& +\sum_{\mathbf{k q} \in \mathbf{S}}|\widehat{r}(\mathbf{k q}, \mathbf{T})-\sqrt{\rho(\mathbf{T})}| \int_{0}^{1} \phi\left(u_{\mathbf{T}}, y, \vartheta_{\mathbf{k}, \mathbf{T}}^{(h)}\right) d h=: \Sigma_{\mathbf{T}, 3}+\Sigma_{\mathbf{T}, 4},
\end{aligned}
$$

where $\theta_{\mathbf{k}, \mathbf{l}}^{(h)}=h r((\mathbf{k q}, \mathbf{l q}))+(1-h) \rho(\mathbf{T})$ and $\vartheta_{\mathbf{k}, \mathbf{T}}^{(h)}=h \widehat{r}((\mathbf{k}, \mathbf{T}))+(1-h) \sqrt{\rho(\mathbf{T})}$. By some computations and Lemma B1 of Tan and Wang (2015), we have for some constant $K>0$

$$
\Sigma_{\mathbf{T}, 3} \leq K \frac{T_{1} T_{2}}{q_{1} q_{2}} \sum_{\mathbf{k q} \in \mathbf{I}_{\mathbf{T}}}|r(\mathbf{k q})-\rho(\mathbf{T})| \exp \left(-\frac{u_{\mathbf{T}}^{2}}{1+\omega_{\mathbf{k q}}}\right) \rightarrow 0
$$

as $\mathbf{T} \rightarrow \infty$, where $\omega_{\mathbf{k q}}=\max \{\rho(\mathbf{T}),|r(\mathbf{k q})|\}$. By the same arguments as for $\Sigma_{\mathbf{n}, 2}$ in (13), we can show that $\Sigma_{\mathbf{T}, 4}=o(1)$, as $\mathbf{T} \rightarrow \infty$.

Fourthly, we prove that as $\varepsilon \downarrow 0$,

$$
\left|P\left(a_{\mathbf{T}}\left(\max _{\mathbf{t} \in \mathfrak{R}\left(q_{1}\right) \times \mathfrak{R}\left(q_{2}\right) \cap \mathbf{S}} \xi_{\mathbf{T}}(\mathbf{t})-b_{\mathbf{T}}\right) \leq x, U \leq y\right)-\int_{-\infty}^{y} \prod_{i_{1}=1}^{n_{1}} \prod_{i_{2}=1}^{n_{2}} P\left(\max _{t \in \mathbf{S}_{\mathbf{i}}} \eta(\mathbf{t}) \leq u_{\mathbf{T}}^{(z)}\right) \phi(z) d z\right| \rightarrow(220)
$$

where

$$
u_{\mathbf{T}}^{(z)}:=\frac{b_{\mathbf{T}}+x / a_{\mathbf{T}}-\rho^{1 / 2}(\mathbf{T}) z}{(1-\rho(\mathbf{T}))^{1 / 2}}=\frac{x+r-\sqrt{2 r} z}{a_{\mathbf{T}}}+b_{\mathbf{T}}+o\left(a_{\mathbf{T}}^{-1}\right) .
$$

By the definition of $\left\{\xi_{\mathbf{T}}(\mathbf{t}), \mathbf{0} \leq \mathbf{t} \leq \mathbf{T}\right\}$, we have

$$
\begin{aligned}
& P\left(a_{\mathbf{T}}\left(\max _{\mathbf{t} \in \mathfrak{R}\left(q_{1}\right) \times \mathfrak{R}\left(q_{2}\right) \cap \mathbf{S}} \xi_{\mathbf{T}}(\mathbf{t})-b_{\mathbf{T}}\right) \leq x, U \leq y\right) \\
& =\int_{-\infty}^{y} P\left(\max _{\mathbf{t} \in \mathfrak{R}\left(q_{1}\right) \times \mathfrak{R}\left(q_{2}\right) \cap \mathbf{S}} \eta(\mathbf{t}) \leq u_{\mathbf{T}}^{(z)}\right) \phi(z) d z \\
& =\int_{-\infty}^{y} \prod_{i_{1}=1}^{n_{1}} \prod_{i_{2}=1}^{n_{2}} P\left(\max _{\mathbf{t} \in \mathfrak{R}\left(q_{1}\right) \times \mathfrak{R}\left(q_{2}\right) \cap \mathbf{S}_{\mathbf{i}}} \eta(\mathbf{t}) \leq u_{\mathbf{T}}^{(z)}\right) \phi(z) d z .
\end{aligned}
$$

As for the discrete case, a direct calculation leads to

$$
u_{\mathbf{T}}^{(z)}=\frac{x+r-\sqrt{2 r} z}{a_{\mathbf{T}}}+b_{\mathbf{T}}+o\left(a_{\mathbf{T}}^{-1}\right) .
$$

Now, by Lemma 4.3 again and the dominated convergence theorem, we have

$$
\mid \int_{-\infty}^{y} \prod_{i_{1}=1}^{n_{1}} \prod_{i_{2}=1}^{n_{2}} P\left(\max _{\mathbf{t} \in \mathfrak{R}\left(q_{1}\right) \times \mathfrak{R}\left(q_{2}\right) \cap \mathbf{S}_{\mathbf{i}}} \eta(\mathbf{t}) \leq u_{\mathbf{T}}^{(z)}\right) \phi(z) d z
$$




$$
-\int_{-\infty}^{y} \prod_{i_{1}=1}^{n_{1}} \prod_{i_{2}=1}^{n_{2}} P\left(\max _{\mathbf{t} \in \mathbf{S}_{\mathbf{i}}} \eta(\mathbf{t}) \leq u_{\mathbf{T}}^{(z)}\right) \phi(z) d z \mid \rightarrow 0
$$

as $\varepsilon \downarrow 0$. Thus, (20) is proved.

By (17)-(20), in order to prove Theorem 3.1, it suffices to show that

$$
\left|\int_{-\infty}^{y} \prod_{i_{1}=1}^{n_{1}} \prod_{i_{2}=1}^{n_{2}} P\left(\max _{\mathbf{t} \in \mathbf{S}_{\mathbf{i}}} \eta(\mathbf{t}) \leq u_{\mathbf{T}}^{(z)}\right) \phi(z) d z-\int_{-\infty}^{y} \exp \left(-e^{-x-r+\sqrt{2 r} z}\right) \phi(z) d z\right| \rightarrow 0
$$

as $\mathbf{T} \rightarrow \infty$.

Using the stationarity of $\{\eta(\mathbf{t}), \mathbf{t} \geq \mathbf{0}\}$ and (7), we have

$$
\begin{aligned}
\prod_{i_{1}=1}^{n_{1}} \prod_{i_{2}=1}^{n_{2}} P\left(\max _{\mathbf{t} \in \mathbf{O}_{\mathbf{i}}} \eta(\mathbf{t}) \leq u_{\mathbf{T}}^{(z)}\right) & =\left[1-P\left(\max _{\mathbf{t} \in \mathbf{S}_{1}} \eta(\mathbf{t})>u_{\mathbf{T}}^{(z)}\right)\right]^{n_{1} n_{2}} \\
& =\exp \left(n_{1} n_{2} \ln \left[1-P\left(\max _{\mathbf{t} \in \mathbf{S}_{1}} \eta(\mathbf{t})>u_{\mathbf{T}}^{(z)}\right)\right]\right) \\
& =\exp \left(-n_{1} n_{2} P\left(\max _{\mathbf{t} \in \mathbf{S}_{1}} \eta(\mathbf{t})>u_{\mathbf{T}}^{(z)}\right)(1+o(1))\right) \\
& =\exp \left(-n_{1} n_{2} \mathcal{H}_{\alpha_{1}} \mathcal{H}_{\alpha_{2}}(1-\delta)^{2}\left(u_{\mathbf{T}}^{(z)}\right)^{2 / \alpha_{1}+2 / \alpha_{2}}\left(1-\Phi\left(u_{\mathbf{T}}^{(z)}\right)\right)(1+o(1))\right) .
\end{aligned}
$$

Now, letting $\delta \rightarrow 0$ and using the definitions of $a_{\mathbf{T}}, b_{\mathbf{T}}$ and $u_{\mathbf{T}}^{(z)}$ we have

$$
n_{1} n_{2} \mathcal{H}_{\alpha_{1}} \mathcal{H}_{\alpha_{2}}(1-\delta)^{2}\left(u_{\mathbf{T}}^{(z)}\right)^{2 / \alpha_{1}+2 / \alpha_{2}}\left(1-\Phi\left(u_{\mathbf{T}}^{(z)}\right)\right) \rightarrow e^{-x-r+\sqrt{2 r} z},
$$

as $\mathbf{T} \rightarrow \infty$, which together with the dominated convergence theorem completes the proof of Theorem 3.1.

Acknowledgement: The authors would like to thank the referee and the editor for the thorough reading and valuable suggestions, which greatly improve the original results of the paper.

\section{References}

[1] Adler, R., Taylor, J.E. 2007. Random Fields and Geometry. New York: Springer.

[2] Anderson, C. W., Turkman, K. F., 1991a. The joint limiting distribution of sums and maxima of stationary sequences. Journal of Applied Probability, 28, 33-44.

[3] Anderson, C. W., Turkman, K. F., 1991b. Sums and maxima in stationary sequences, Journal of Applied Probability, 28, 715-716.

[4] Berman, S.M., 1974. Sojourns and extremes of Gaussian processes, Ann. Probab., 2, 999-1026.

[5] Choi, H., 2002. Central limit theory and extemes of random fields. Phd Dissertation in Univ. of North Carolina at Chapel Hill.

[6] Choi, H., 2010. Almost sure limit theorem for stationary Gaussian random fields. Journal of the Korean Statistical Society, 39, 449-454.

[7] Chow, T. L., Teugels, J. L., 1979. The sum and the maximum of i.i.d. random variables. In: Proceedings of the Second Prague Symposium on Asymptotic Statistics (Hradec Kralove, 1978), North-Holland, New York, 81-92.

[8] Dȩbicki, K., Hashorva, E., Soja-Kukiela, N., 2015. Extremes of homogeneous Gaussian random fields, Journal of Applied Probability, 52, 55-67.

[9] Ferreira, H., Pereira, L., 2008. How to compute the extremal index of stationary random fields. Statistics and Probability Letters, 78, 1301-1304.

[10] Ferreira, H., Pereira, L., 2012. Point processes of exceedances by random fields. Journal of Statistical Planning and Inference, 142, 773-779.

[11] Ho, H. C., McCormick, W. P., 1999. Asymptotic distribution of sum and maximum for strongly dependent Gaussian processes. Journal of Applied Probability, 36, 1031-1044. 
[12] Hsing, T., 1995. A note on the asymptotic independence of the sum and maximum of strongly mixing stationary random variables. Ann. Probab., 23, 938-947.

[13] Leadbetter, M. R., Lindgren, G. and Rootzén, H., 1983. Extremes and Related Properties of Random Sequences and Processes. New York: Springer-Verlag.

[14] Mathew, G., McCormick, W. P., 1993. A complete Poisson convergence result for a strongly dependent isotropic Gaussian random field. Communications in Statistics Stochastic Models, 9, 13-29.

[15] McCormick, W. P., 1978. Weak and strong laws for the maxima of stationary Gaussian processes. Phd Dissertation in Stanford University at California.

[16] McCormick, W. P., Qi, Y. C., 2000. Asymptotic distribution for the sum and the maximum of Gaussian processes. Journal of Applied Probability, 37, 958-971.

[17] McCormick, W. P., Mittal, Y., 1976. On weak convergence of the maximum. Tech. Report No 81, Dept. of Statist. Stanford University.

[18] Mittal, Y., Ylvisaker, D., 1975. Limit distribution for the maximum of stationary Gaussian processes. Stochastic Process. Appl., 3, 1-18.

[19] Mittal, Y., 1976. A class of isotropic covariances functions. Pacific J. Math. 64, 517-538.

[20] Peng, Z. X., Nadarajah, S., 2002. On the joint limiting distribution of sums and maxima of stationary normal sequence. Theory Probability and Its Application, 47, 817-820.

[21] Pereira, L., 2009. The asymptotic location of the maximum of a stationary random field. Statistics and Probability Letters, 79, 2166-2169.

[22] Pereira, L., 2010. On the extremal behavior of a nonstationary normal random field. Journal of Statistical Planning and Inference, 140, 3567-3576.

[23] Pereira, L., Ferreira, H., 2006. Limiting crossing probabilities of random fields. Journal of Applied Probability, 43, 884-891.

[24] Pereira, L., Tan, Z., 2016. Almost sure convergence for the maximum of nonstationary random fields, Journal of Theorotical Probablity, doi 10.1007/s10959-015-0663-3, (In press).

[25] Piterbarg, V.I., 1996. Asymptotic Methods in the Theory of Gaussian Processes and Fields. AMS, Providence.

[26] Tan, Z., 2013. The limit theorems on extremes for Gaussian random fields, Statistics and Probability Letters, $83,436-444$.

[27] Tan, Z., Wang, K., 2015. On Piterbarg's max-discretisation theorem for homogeneous Gaussian random fields, Journal of Mathematical Analysis and Application, 429, 969-994.

[28] Tan, Z., Wang, Y., 2014. Almost sure asymptotics for extremes of non-stationary Gaussian random fields. Chinese Annals of Mathematics, Series B, 35, 125-138.

[29] Tan, Z., Yang, Y., 2015. The maxima and sums of multivariate non-stationary Gaussian sequences. Appl. Math. J. Chinese Univ., 30, 197-209. 